Journal of Computer Science 5 (10): 732-737, 2009

ISSN 1549-3636

(C) 2009 Science Publications

\title{
Performance Evaluation of Stable Weight-Based on Demand Routing Protocol for Mobile Ad Hoc Network
}

\author{
Oon Chong Hsi and Azizol Abdullah \\ Department of Communication Technology and Network, \\ Faculty of Computer Science and Information Technology, \\ University Putra Malaysia, 43400 UPM, Serdang, Selangor, Malaysia
}

\begin{abstract}
Problem statement: A MANET is an autonomous collection of mobile users that communicate over relatively bandwidth constrained wireless links. Since the nodes are mobile, the network topology may change rapidly and unpredictably over time. Approach: A Stable Weight-based On demand Routing Protocol (SWORP) that uses the weight-based route strategy to select a stable route was created by Wang. But SWORP only evaluated in a limited setting of simulation, more simulation parameter have to test with SWORP to evaluate how far this protocol can go on. In this project, SWORP was implemented in simulation environment with two other routing protocols, AODV and DSR. Results: These three protocols were implemented in Network Simulator 2 (NS2) and the performance was compare with performance metrics, end-to-end delay, number of packet drop and packet delivery ratio. Conclusion: As expected, SWORP had outperformed AODV and DSR in the overall routing performance.
\end{abstract}

Key words: Mobile ad hoc network, on-demand routing protocol, on-demand routing, weight-based routing, stable route

\section{INTRODUCTION}

A wireless ad hoc network is a dynamic network consisted of a group of mobile device in which communicate with each other by wireless media. Communication can be done when a node is in the wireless transmission region of another node. A source can send data to a destination which is not in its communication region through a group of nodes that willing to forward its packets. The determination of which nodes forward data is made dynamically based on the network connectivity. This is in contrast to wired networks in which routers perform the task of routing. It is also in contrast to managed wireless networks, in which a special node known as an access point manages communication among other nodes.

The other type of mobile wireless network is the non-infrastructure network commonly known as Mobile Ad hoc Network (MANET). A MANET is an autonomous collection of mobile users that communicate over relatively bandwidth constrained wireless links. Since the nodes are mobile, the network topology may change rapidly and unpredictably over time. From the article Mobile ad hoc network of
National Institute of Standards and Technology, they state that the network is decentralized, where all network activity including discovering the topology and delivering messages must be executed by the nodes themselves. Different protocols are then evaluated based on the packet drop rate, the overhead introduced by the routing protocol and other measures.

In MANET, the main issues are bandwidth, Quality of Service (QoS) and mobility. Mobile nodes cooperate to forward packets over the MANET with a broadcast technique call blind flooding. With blind flooding, each node will rebroadcast the packet whenever it receives the packet for the first time and consume lots of bandwidth. QoS is very important since multimedia service have become popular. Over the past few years, there have been a considerable number of studies on $\mathrm{QoS}^{[1]}$. In a mobile environment, because of the mobility of mobile nodes in MANETs, the shortest path is not necessarily the best path. If the stability of a routing path is not considered, then wireless links may be easily broken. Many efforts have been made to design reliable routing protocols that enhance network stability. Wang ${ }^{[1]}$ had proposed a Stable Weight-based On Demand Routing Protocol (SWORP) to overcome

Corresponding Author: Azizol Abdullah, Department of Communication Technology and Network,

Faculty of Computer Science and Information Technology, University Putra Malaysia,

43400 UPM Serdang, Selangor, Malaysia 
the problem of route stability with choosing the most stable route according to its weight selection algorithm. The result of the proposed SWORP was only evaluated by Wang himself, more simulation experiment should be done in this protocol to evaluate the potential of this protocol.

In this study, we evaluate SWORP performance with Ad-hoc On-demand Distance Vector Routing (AODV) and Dynamic Source Routing (DSR) performance in a simulated environment using other useful parameters such as end-to-end delay and number of packet drop.

Ad hoc routing protocol: The following sections introduce two famous ad hoc routing protocols, AODV and DSR. This two routing protocol have been widely used in wireless research area as the benchmark of the research.

Ad hoc On-demand Distance Vector (AODV): Adhoc On-demand Distance Vector (AODV) routing algorithm is an algorithm for routing data across Wireless Mesh Networks. It is capable of both unicast and multicast routing. It is a reactive routing protocol, meaning that it establishes a route to a destination only on demand. In AODV, each mobile node discovers or maintains routing information to another node if it is actively communicating with that node, or if it is intermediary between two end points. If a node does not lie on an active path between two nodes, it does not maintain routing information for that path. AODV dynamically maintains loop-free routes, even when links change on active routes.

AODV routing protocol was first proposed in IETF Internet draft in fall of 1997. Since that first version, AODV has evolved into a carefully specified ad hoc network routing protocol that provide path discovery and maintenance in a wide variety of network topologies and environments. AODV has been researched both by its original authors and by numerous other researchers within the mobile networking community ${ }^{[2]}$.

Mobility Management and Networking Laboratory have state the process of AODV's path selection process. AODV builds routes using a route request (RREQ)/route reply (RREP) query cycle. When a source node desires a route to a destination for which it does not already have a route, it broadcasts a RREQ packet across the network. Nodes receiving this packet update their information for the source node and set up backwards pointers to the source node in the route tables. In addition to the source node's Internet Protocol (IP) address, current sequence number and broadcast
Identity (ID), the RREQ also contains the most recent sequence number for the destination of which the source node is aware. A node receiving the RREQ may send a RREP if it is either the destination or if it has a route to the destination with corresponding sequence number greater than or equal to that contained in the RREQ. If this is the case, it wills unicast a RREP back to the source. Otherwise, it rebroadcasts the RREQ. Nodes keep track of the RREQ's source IP address and broadcast ID. If they receive a RREQ which they have already processed, they discard the RREQ and do not forward it.

As the RREP propagates back to the source, node will set up forward pointers to the destination. Once the source node receives the RREP, it may begin to forward data packets to the destination. If the source later receives a RREP containing a greater sequence number or contains the same sequence number with a smaller hop count, it may update its routing information for that destination and begin using the better route.

As long as the route remains active, it will continue to be maintained. A route is considered active as long as there are data packets periodically travelling from the source to the destination along that path. Once the source stops sending data packets, the links will time out and eventually be deleted from the intermediate node routing tables. If a link break occurs while the route is active, the node upstream of the break propagates a route error (RERR) message to the source node to inform it of the now unreachable destination. After receiving the RERR, if the source node still desires the route, it can reinitiate route discovery.

Dynamic Source Routing (DSR): Dynamic Source Routing (DSR) is a routing protocol for wireless mesh networks. It is similar to AODV in that it forms a route on-demand when a transmitting computer requests one. However, it uses source routing instead of relying on the routing table at each intermediate device.

Determining source routes requires accumulating the address of each device between the source and destination during route discovery. The accumulated path information is cached by nodes processing the route discovery packets. The learned paths are used to route packets. To accomplish source routing, the routed packets contain the address of each device the packet will traverse. This may result in high overhead for long paths or large addresses, like IPv6. To avoid using source routing, DSR optionally defines a flow id option that allows packets to be forwarded on a hop-by-hop basis. This protocol is truly based on source routing whereby all the routing information is maintained (continually updated) at mobile nodes. 


\section{MATERIALS AND METHODS}

Stable Weight-based On-demand Routing Protocol: (SWORP) The basic idea of SWORP is to select a stable routing path for routing to reduce the routing overhead and packet loss. Several parameters were defined in SWORP as below:

- Duration of Time (DT): The minimal value of the error count for each node in a path

- Error Count (EC): The maximal value of the error count for each node in a path

- Hop Count (HC): The number of hops in a path

- MaxDT: The maximum value of the duration of time all feasible paths

- MaxEC: The maximum value of the error counts regarding all feasible paths

- MaxHC: The maximum value of the hop counts regarding all feasible paths

Route discovery: SWORP is an on-demand routing protocol and the route discovery is similar to that of the DSR. The route discovery process is initiated whenever a source node wants to communicate with other node, for which it has no routing information in its table. The source node initiates route discovery by broadcasting a RREQ packet to its neighboring nodes. Every node maintains two separate counters: A node sequence number and a routing list ID.

The pair < Source ID, Routing List ID> uniquely identifies a route request. Routing list ID is incremented whenever the source node issues a new route request. Each node had received RREQ. First, check whether it knows of a route to the destination in their route table. If it exists, then check its sequence number to see whether it is greater than destination sequence number. If the two conditions are satisfied, then the intermediate node sends a route discovery response along the reverse path back to the node. Otherwise, the node increment the hop count by one and rebroadcasts the route discovery packet. Note that a node may receive multiple copies of the same RREQ packet form various neighboring nodes. When an intermediate node receives a RREQ packet with the same source ID and routing list ID, it discards the redundant RREQ packet and does not rebroadcast it. When the destination node finds a suitable path, it sends back a RREP packet to the source node.

By the time a broadcast packet arrives at a node that can supply a route to the destination node, a reverse path has been established to the source node of the route request. As the RREP packet travels back to the source node, each node along the path sets up a forward pointer to the node from which the route reply came, updates its timeout information for route entries to the source node and the destination node and records the latest destination sequence number for the requested destination node.

The detailed route discovery process and the steps of route discovery are described in two steps below:

- The source node $\mathrm{S}$ broadcasts a RREQ packet to its neighboring nodes. If the node is in the transmission radius, it forwards the RREQ packet to its own neighboring nodes and adds its ID, the hop count, the error count and the duration of time of the RREQ packet to the packet entry

- When the destination node D receives a RREQ packet, it waits for a certain time to receive other RREQ packets. Then node D computes the weight value using weight function. Afterwards node $\mathrm{D}$ selects the path with the maximum weight value as the main data transmission routing path among all feasible paths. Finally node D sends a RREP packet to source node $\mathrm{S}$ along the main routing path

Routing weight function: An algorithm which effectively combines all the three parameters with certain weighing factor $C_{1}, C_{2}$ and $C_{3}$ were proposed. The value of the factor can be chosen according to the system needs. The flexibility of changing the factors helps $\mathrm{n}$ applying our algorithm to select routing path. Larger duration of time represents higher reliability and so do lower error count and lower hop count.

The weight function is defined as an empirical mean value, where we first normalize each item and then combine these three quantities. More precisely:

$\mathrm{W}_{\mathrm{i}}=\mathrm{C}_{1} \times\left(\frac{\mathrm{DT}_{\mathrm{i}}}{\mathrm{MaxDT}}\right)+\mathrm{C}_{2} \times\left(\frac{\mathrm{EC}_{\mathrm{i}}}{\mathrm{MaxEC}}\right)+\mathrm{C}_{3} \times\left(\frac{\mathrm{HC}_{\mathrm{i}}}{\mathrm{MaxHC}}\right)$

where, $\left|\mathrm{C}_{1}\right|+\left|\mathrm{C}_{2}\right|+\left|\mathrm{C}_{3}\right|=1$.

Route maintenance: Due to the high mobility of nodes in MANETs, links break easily. SWORP route maintenance is initiated while the route is active and data packets are transmitting. A link failure occurs when a mobile node that discovers link failure broadcast a RERR packet to other mobile nodes. Mobile nodes that received the RERR packets find out this malefactor in their route cache and add one to its error count. On receiving a RERR packet, the source 
node initiates a new route discovery or finds an alternative path for routing.

The detailed process and the steps of the route maintenance are described below:

- Assume that node $\mathrm{I}$ is chosen to be the transmitting node. When a link is broken, node I receives a RERR packet

- $\quad$ Node I send the RERR packet to the source node S. When the source node $S$ receives RERR packet, the source node $\mathrm{S}$ stops to send the data and then restarts the route discovery process or finds an alternative path for routing

\section{RESULTS AND DISCUSSION}

Performance evaluation: We decided to use a simulator for our performance study because a practical implementation of an ad hoc network was obviously not feasible. We chose the popular network simulator NS2 as the simulator primarily to implement methods because it is widespread use in the academic community and the comprehensive manuals and tutorials that are freely available. It is possible to simulate a mobile multi-hop ad hoc wireless network in ns-2 using simulated 802.11 MAC layer.

As shown in Table 1, we first made some assumptions on the parameters of the system architecture in the simulations.

The simulation modeled a network in a $600 \times 600 \mathrm{~m}$ area with 40-120 mobile nodes. The radio transmission range for each node was assumed to be $100 \mathrm{~m}$. The speed of each mobile node was assumed varied from 4$13 \mathrm{~m} \mathrm{sec}^{-1}$.

In these simulations used the same communication pattern for all mobility simulations. The traffic pattern consisted of Constant Bit Rate (CBR) traffic type. In these simulation did not use TCP because did not want to investigate TCP, which uses flow control, retransmit features and so on. The communication traffic and scenarios simulations are randomly generated by NS2 itself. We assumed that the mobility of the mobile nodes was random. Each simulation was run for $600 \mathrm{sec}$.

There was three performance metrics used to evaluate the performance of SWORP, AODV and DSR in this project. The performance metrics used was described below:

- End-to-end delay: The time it takes for a packet to be sent by the source node to the destination node

- Number of packet drop: The total number of packets dropped during routing
- Packet delivery ratio: The ratio of the number of data packets received by a destination over the number of data packets delivered by the corresponding source

These metrics was chosen due to these performance metrics was well known in showing the routing performance of the selected protocol.

End-to-end delay: Figure 1 and 2 show the end-to-end delay of SWORP, AODV and DSR with different number of mobile nodes and mobility speeds, respectively. As shown in Fig. 1 and 2, the end-to-end delay increases as the number of mobile nodes or the mobility speed increases. The end-to-end delay for SWORP is higher than AODV and DSR. This is because SWORP have extra mechanism to select a path and it is also possible that SWORP select a longer hop route since it has to consider the stability problem. In addition, AODV and DSR only select the shortest path or fastest transmission path.

Number of packet drop: Figure 3 and 4 shows a comparison of the number of packets dropped of SWORP, AODV and DSR with different number of mobile nodes and mobility speeds, respectively. Similar to Fig. 1 and 2, the number of packets dropped increases as the number of mobile nodes or the mobility speed increases.

Table 1: Parameter and used during simulation

\begin{tabular}{ll}
\hline Parameters & Value \\
\hline Area & $600 \times 600 \mathrm{~m}$ \\
No. of nodes & $40-120$ \\
Simulation duration & $600 \mathrm{sec}$ \\
No. of repetition & 6 times \\
Radio transmission range & $100 \mathrm{~m}$ \\
Physical/Mac layer & IEEE 802 \\
Pause time & $100 \mathrm{sec}$ \\
Mobility model & Random waypoint model \\
Node movement & $4-13 \mathrm{~m} \mathrm{sec}^{-1}$ \\
Data sending rate & $2 \mathrm{kbps}$ \\
Each packet & 2 mega byte \\
\hline
\end{tabular}

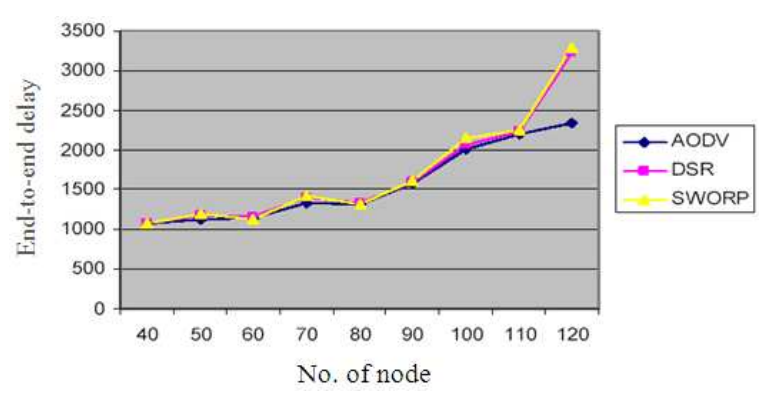

Fig. 1: End-to-end delay versus number of nodes with $10 \mathrm{~m} \mathrm{sec}^{-1}$ 


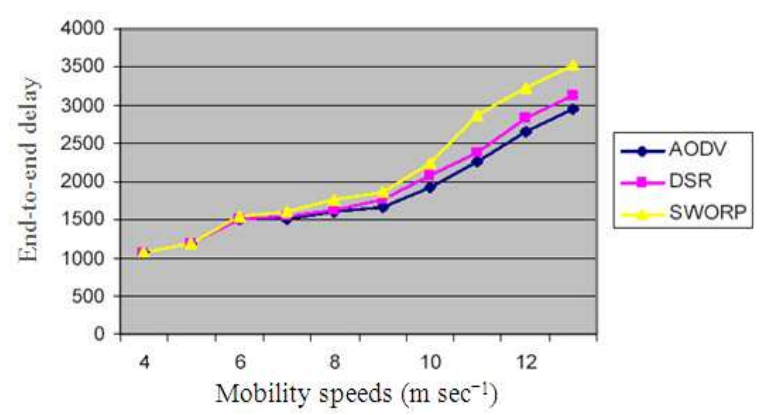

Fig. 2: End-to-end delay versus mobility speeds with 50 nodes

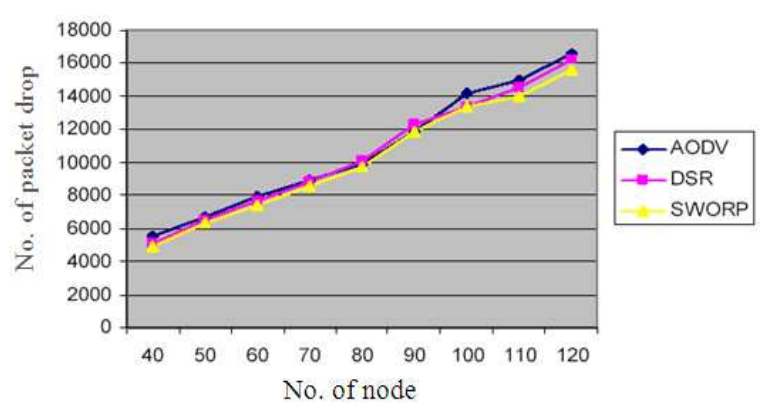

Fig. 3: Number of packet drop versus number of nodes with $10 \mathrm{~m} \mathrm{sec}^{-1}$

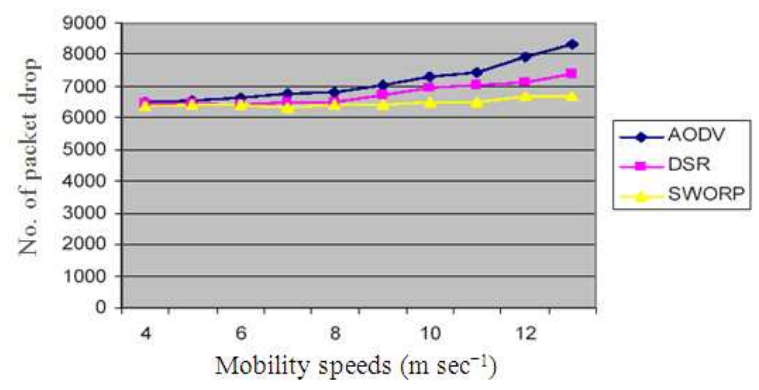

Fig. 4: Number of packet drop versus mobility speeds with 50 nodes

Packet delivery ratio: Figure 5 and 6 show the packet delivery ratio of SWORP, AODV and DSR with different number of mobile nodes and mobility speeds, respectively. We can observe that SWORP transmits and receives more data packet than AODV and DSR. This is because AODV and DSR path may broken easily, but SWORP will always choose the most stable path, the chance of link breakage for SWORP is lower than AODV and DSR since AODV and DSR didn't consider the stability of the selected path.

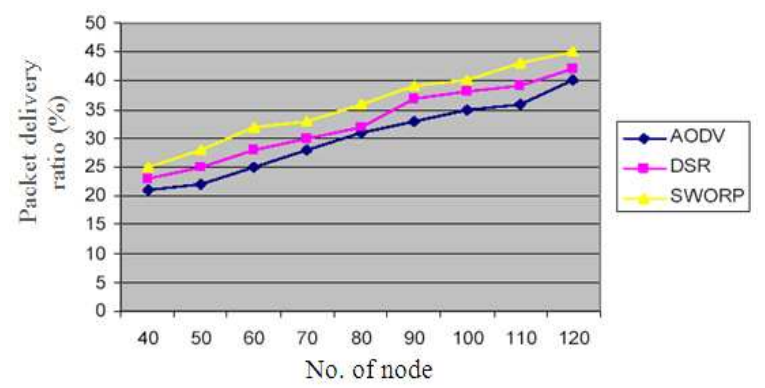

Fig. 5: Packet delivery ratio versus number of nodes with $10 \mathrm{~m} \mathrm{sec}^{-1}$

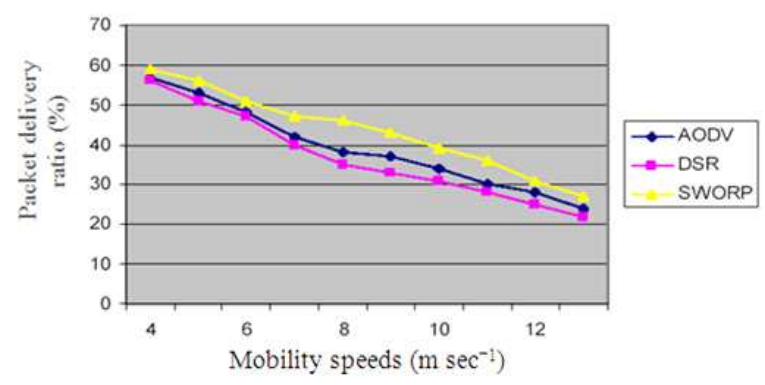

Fig. 6: Packet delivery ratio versus mobility speeds with 50 nodes

Because of SWORP always choose the most stable route for transmission that reduces the number of packets dropped, so the number of call dropping of SWORP is lower than that of AODV and DSR.

\section{CONCLUSION}

The objective of this project is to build SWORP and compare its performance with other well known on demand ad hoc protocol, AODV and DSR. These three protocols were evaluated through NS2.

The main differences between SWORP and other on demand routing protocol is that SWORP selects a stable routing path by maximizing the weight among the feasible path. The three important parameters used in SWORP to measure the weight of the feasible path is the duration of time, the error count and hop count. The route selection is based on the weight value of each feasible path. In a feasible path, the less weigh value represents less reliability. It also represents higher mobility of each node in the path. SWORP always select the most stable path for routing. Experimental results show that the SWORP was outperforms DSR and AODV especially in the high mobility environment. 
The high performance of SWORP has makes it become a trustable and adaptable to wireless ad hoc activity. However, there is still a lot of work to be done concerning this protocol and further studies could be conducted concerning them. The performance of SWORP can be compare with other newer protocol to test if it is suitable for nowadays environment. SWORP also can be test in different mobility model to test whether it is suitable for that mobility model.

\section{REFERENCES}

1. Wang, N.C., Y.F. Huang and J.C. Chen, 2007. A stable weight-based on-demand routing protocol for mobile ad hoc networks. Inform. Sci., 177: 5522-5537.

http://portal.acm.org/citation.cfm?id=1293616

2. Chandrashekar, K., 2003. Multicast Routing in Mobile Ad hoc Networks using source grouped flooding. M.Sc. Thesis, University of Maryland. http://www.stormingmedia.us/33/3381/A338144.html

3. Mohan, P.M., J.J. Johnson, K. Murugan and V. Ramachandran, 2001. A comparative and performance study of on demand multicast routing protocols for ad hoc networks. College of Engineering, Guindy (CEG), Anna University. http://www.hipc.org/hipc2002/2002Posters/OnDe mandMulticast.pdf

4. West, D., 2003. An implementation and evaluation of the ad-hoc on-demand distance vector routing protocol for windows CE. M.Sc. Thesis, University of Dublin. http://www.cs.tcd.ie/publications/techreports/reports.03/TCD-CS-2003-61.pdf
5. Rasheed, T.M., L. Reynaud, Y. Gourhant and K. Al Agha, 2006. Reliability of weight metric based clustering models in large scale mobile ad hoc networks. Proceeding of the Wireless Networks and Emerging Technology, July 3-5, Banff, Canada, pp: 572.

http://www.actapress.com/PaperInfo.aspx?PaperID $=27433 \&$ reason $=500$

6. Mainak, C., K.D. Sajal and T. Damla, 2000. A weight based distributed clustering algorithm for mobile ad hoc network. Proceeding of the 7th International Conference on High Performance Computing, Dec. 17-20, Amsterdam, Netherlands, pp: 511-521.

http://portal.acm.org/citation.cfm?id=653181

7. National Institute of Standards and Technology, 2001. Mobile Ad Hoc Network (MANET). http://w3.antd.nist.gov/wahn_mahn.shtml

8. Mobility Management and Networking Laboratory, 2002. The Ad hoc On Demand Distance Vector (AODV). http://moment.cs.ucsb.edu/AODV/

9. Corson, S., 1999. Mobile Ad Hoc Network (MANET) reference draft. http://www.ietf.org/rfc/rfc2501.txt 